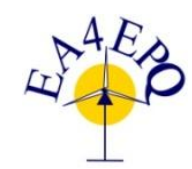

International Conference on Renewable Energies and Power Quality (ICREPQ'13) Bilbao (Spain), 20 ${ }^{\text {th }}$ to $22^{\text {th }}$ March, 2013

Renewable Energy and Pourer Qualily. Tournal (RE\&PQJ)

ISSN 2172-038 X, No.11, March 2013

\title{
Analysis of PRIME PLC Smart Metering Networks Performance
} \author{
Ferro Vázquez ${ }^{1}$ \\ ${ }^{1}$ Telecommunications Department \\ Iberdrola Engineering and Construction S:A.U. \\ Ribera de Axpe, 5 - 48950 Erandio (Spain) \\ afol@iberdrola.es, jmae@iberdrola.es,jfv@iberdrola.es \\ ${ }^{2}$ Control Systems and Telecommunications Division \\ Iberdrola Networks \\ Avda. San Adrian, 48 - 48003 Bilbao (Spain) \\ asendin@iberdrola.es, iuga@iberdrola.es \\ ${ }^{3}$ Electronics and Telecommunications Department \\ University of the Basque Country (UPV/EHU) \\ Alda. Urquijo, S/N - 48013 Bilbao (Spain) \\ pablo.angueira@ehu.es
}

A. Fernandez Olivera ${ }^{1}$, A. Sendin Escalona ${ }^{2}$, I. Urrutia Galdos ${ }^{2}$, J. Mateo Arenas ${ }^{1}$, P. Angueira Buceta ${ }^{3}$ and JJ.

\begin{abstract}
This paper describes the process followed to assess the performance of Smart Metering PLC networks based on PRIME specification. This process is composed of a monitoring phase dedicated to obtain data from the network (Communication level and Application level), and an analysis phase which produces a standardized report for each Secondary Substation with the relevant performance data correlated with electricity grid information. The paper includes also a section identifying different specific abnormal behaviours in PLC Smart Meters, working in the grid.
\end{abstract}

\section{Key words}

Smart Metering, PRIME, DLMS, PLC, Analysis

\section{Introduction}

The Spanish Royal Decree 809/2006, published in June 2006 mandated that, as from $1^{\text {st }}$ July 2007, all meters to be installed in new homes should include time-of-use functionality and remote management capabilities [1]. One year later, the Spanish order, ITC/3860/2007 [2] forced a massive replacement of all residential meters before $31^{\text {st }}$ of December 2018. Further information to this replacement is included in the IET/290/2012 [3] which describes the milestones that must be fulfilled during the implementation phase.

The new meters are called Smart Meters and are part of the electricity grid known as Smart Grid. This type of meters is the first step in the adaptation of the electricity grid towards the future grid, with a highly automated operation and a vast amount of monitored data.

Power Line Communications (PLC) is one of the preferred technologies for Smart Metering [4-7]. This technology uses the electric power lines in order to deploy telecommunications. PLC offers low cost, ease of deployment and scalability. The same grid used for energy distribution provides the basis for smart metering communication, eliminating the need for additional communications infrastructure and allowing the communication network to grow together with the distribution grid.

One example of a PLC technology used in low voltage grid is PRIME, a narrowband data transmission system technology based on Orthogonal Frequency Division Multiplexing (OFDM), in frequencies defined by CENELEC A (between $42 \mathrm{kHz}$ to $90 \mathrm{kHz}$ ) [8]. It uses three modulation schemes (DBPSK, DQPSK and D8PSK, with or without forward error correction) [9] with a physical layer capable of achieving rates of uncoded 128kbps, a "plug and play" MAC layer optimised for the power line environment, a convergence layer for IP and a convergence layer for IEC 61334-4-32 [10 - 11].

This paper is organized as follows. Section 2 introduces the PRIME technology oriented towards Smart Metering. Section 3 describes how the PLC technology is deployed on a Smart Grid consisting of a data concentrator and smart meters in each subnetwork. Section 4 shows the importance of testing the grid before production environment. Section 5 explains the tool used to monitor the Smart Grid and how the results are analyzed. Section 6 specifies some special features of the analysis and Section 7 summarizes the conclusions.

\section{PRIME technology description}

PRIME technology defines two types of nodes: one Base Node and Service Nodes. The Base Node is the core of the system, and it is also referred to as Data Concentrator or, simply, Concentrator. This node is normally connected at the Secondary Substation (SS) and it is responsible for the control of the subnetwork including all Service Nodes (meters in the application 
layer considered in this paper) connected to it. Each node that takes part in the Subnetwork is called Service Node. The Service Node may operate in three different states (Figure 1).

- Disconnected: The starting point of every Service Node. In this state the meter is not yet registered in the subnetwork.

- Terminal: Once the Service Node is registered into the network, changes to Terminal state and it can start communicating.

- Switch: Service Nodes that are able to provide communications access to other meters that can also be switches or terminals.

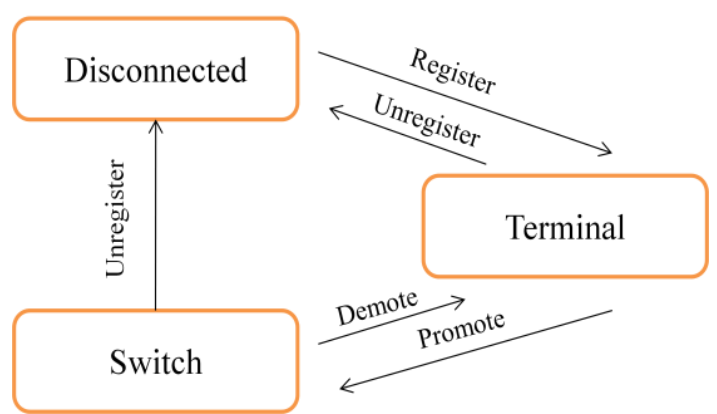

Figure. 1. State machine of a Service Node.

When a Service Node is a Switch, it acts as a repeater of the information exchanged between the Base Node and any Service Node connected to the subnetwork through this Switch. In the subnetwork, each Switch is identified with an identification number called SID.

Service Nodes change their state depending on the grid conditions (attenuation, noise) in real time, and they may be in different states during a certain period, as the subnetwork is dynamic.

The communication relationship among the Base Node and the Service Nodes is Master / Slave.

PRIME is a technology that allows real time monitoring of Smart Metering networks. The main monitored concept is meter availability. This parameter shows when a meter is ready to communicate in a subnetwork, and is based on PRIME state machine (Figure 1).

\section{Deployment of PLC technology}

PRIME uses the electricity grid to create and maintain the topology of the subnetworks. As the network elements are plug and play, the deployment is straight-forward in all cases. Figure 2 shows a typical topology of electricity grid elements for PRIME. CGP is the Spanish acronym for Fuse Box that According to Spanish REBT [12], is the border in LV grid property between the utility and the end users.

One of the advantages of PRIME is that a wide variety of manufacturers offer products compliant with the specification, as guaranteed by a certification process inside PRIME Alliance [13]. Based on this, there is a fullfledged ecosystem with enough competence and competitors for the electricity companies.

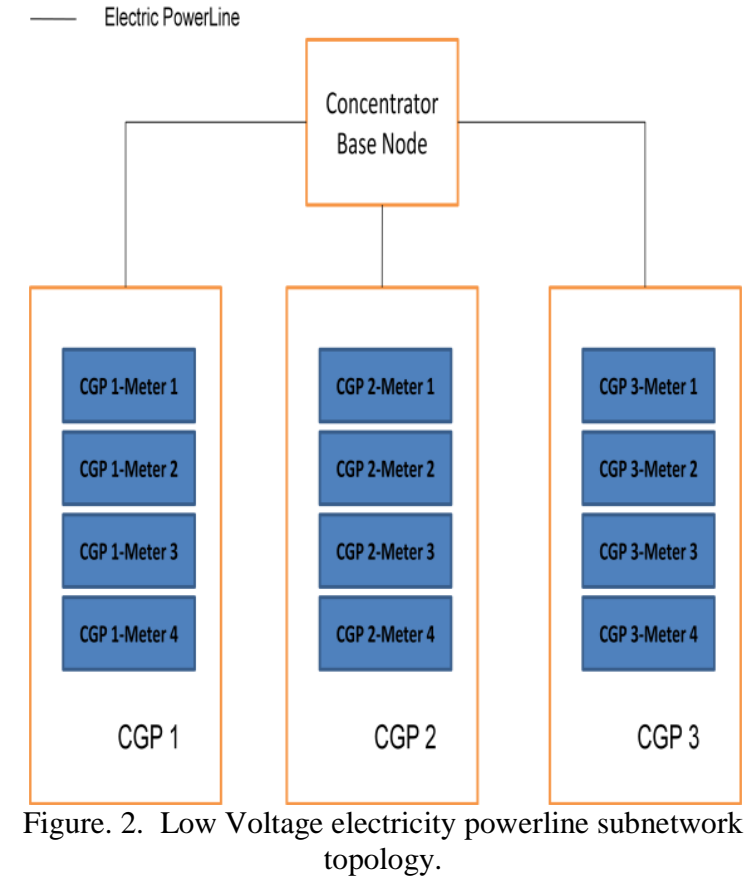

The deployment in Iberdrola has proven that the technology is performing successfully and, as of today, more than 3,500 SS's, with more than 700,000 PRIME smart meters, are assessed using the analysis tool explained in this paper.

\section{The importance of the analysis during massive deployment in PRIME}

In a perfect world (e.g., a laboratory installation), where all the elements of the subnetwork work without errors, it is not necessary to analyze the behaviour of smart meters. However, it is very common in field installations to find unexpected behaviours, and problems related to the grid conditions themselves or to the different operation and maintenance problems that affect to any electronic and / or telecommunications device.

Classification of common problems can be found in Table I. It is important to clarify that the nomenclature of the devices depends on the protocol layer considered. In PRIME layer, as it is mentioned in Section 2, the devices are called Service Nodes and Base Node, but in the application layer (DLMS protocol in smart metering networks), these devices are called meters and concentrator respectively.

The following list shows usual behaviours found during the test analysis:

1) Meters not found in the Subnetwork.

2) Meters found in a different SS than expected.

3) Meters registered in PRIME subnetwork but not functional at application level.

4) Meters not found in any network. 
Table I. - Classification of problems for Smart Metering [14

\begin{tabular}{|c|c|}
\hline Environment & $\begin{array}{r}\text { Problems } \\
\end{array}$ \\
\hline $\begin{array}{l}\text { Electricity } \\
\text { Network }\end{array}$ & $\begin{array}{l}\text { Devices not correctly assigned in } \\
\text { databases (electricity grid likely structure, } \\
\ldots \text {..). } \\
\text { Electricity infrastructure related problems } \\
\text { (transformer, LV panel, electricity line, } \\
\text { phase,...). }\end{array}$ \\
\hline PLC Network & $\begin{array}{l}\text { Devices not communicating permanently. } \\
\text { Devices not communicating occasionally } \\
\text { (noise events, ...). } \\
\text { Devices communicating through different } \\
\text { Base Nodes. }\end{array}$ \\
\hline $\begin{array}{l}\text { Metering } \\
\text { Network }\end{array}$ & $\begin{array}{l}\text { Devices not working properly from an } \\
\text { application level perspective (for some } \\
\text { type of queries, reports, ...). }\end{array}$ \\
\hline Device Faults & $\begin{array}{l}\text { Permanent failures. } \\
\text { Progressive failures. }\end{array}$ \\
\hline
\end{tabular}

These behaviours are usually caused by the problems explained in Table I and generate performance issues in the meters. Bad implementations of PRIME protocol may also cause performance problems, and they must be considered during the analysis.

All these problems should be solved before the Smart Meters are connected to Meter Data Management (MDM) systems. This is the reason why stress tests and analysis are mandated and performed in Iberdrola for all deployed SS's.

\section{Description of the analysis of PRIME Smart Meters networks}

Using the tool mentioned in [14], every SS deployed with Smart Meters goes through a monitoring and analysis process that is oriented to assess its performance, and to identify operational problems. The tool implements a methodology specifically developed for this purpose.

The monitoring stage gathers all the information needed to assess the performance during a one week period, both from the PRIME communication level, and the application level (DLMS / COSEM). The analysis stage performs the assessment of the results, based on the correlation of all data, together with basic electricity infrastructure.

A typical report generated by the tool contains the following information:

1) PRIME subnetwork evolution. It is a graph with the evolution of the registered nodes in the subnetwork. It is also important to have the same graph with the evolution of the switches (see Figure 3).

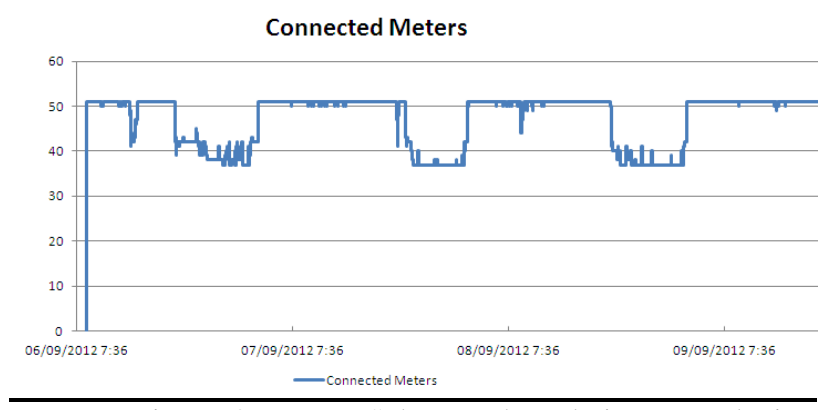

Figure. 3. PRIME Subnetwork evolution example, in an exceptional noisy grid

2) PRIME availability of each meter. It is considered in terms of percentage of time.

3) PRIME availability of meters identified by its situation in the electrical network (see Figure 4).

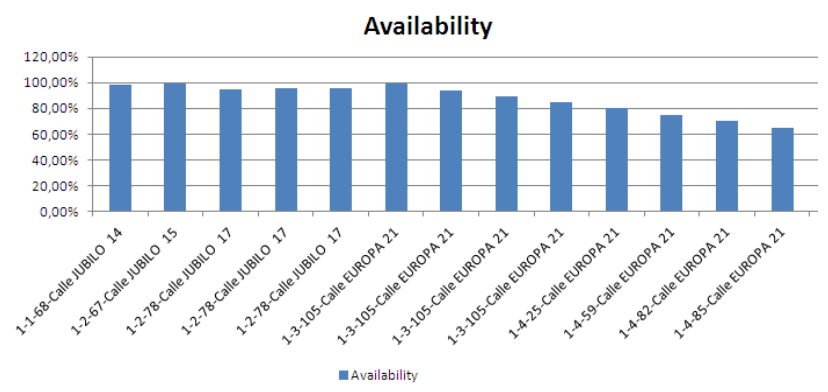

Figure. 4. PRIME Availability order by its situation

4) PRIME hierarchy of the subnetwork. It is a tool to generate a hierarchy map between the concentrator and meters. It shows if a meter is in terminal or switch state and, in case of being in switch state, what meters communicate with it. It is presented both as the instantaneous situation, and the most common situations.

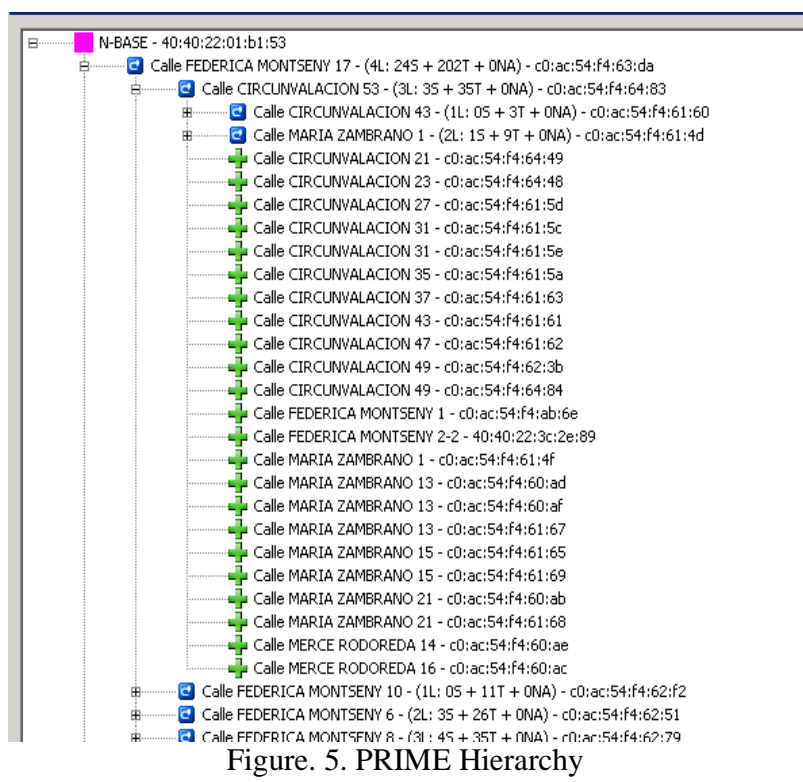


5) PRIME availability of meter concentrations.

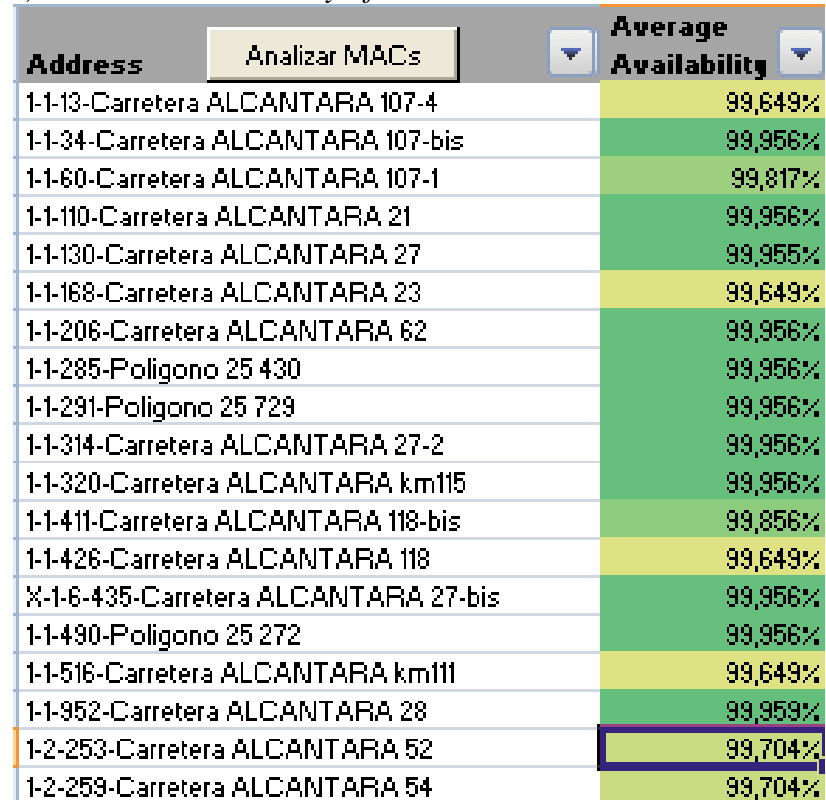

Figure. 6. PRIME availability of meter concentrations example

6) Application level evolution: It is a graph with the evolution of the successes in the different simulated measures.

7) Application level availability. It is considered in terms of percentage of successful transactions at DLMS Cosem level (load curve, measures etc.) between the concentrator and each meter.Depending on the number of meters associated to the concentrator, the number of transactions attempted will be different in the same period of time.

Once the result is generated, there are important points that have to be checked to confirm the good health of a particular subnetwork. Following is a list of the problems that are detected with this information:

PRIME level:

- General problem of the subnetwork, normally caused by a noise or by a bad physical installation. Analysing the evolution of the number of connected nodes using the graph created with the tool, it is easy to see that the network does not manage to register every node. If it is due to a periodic noise, we will see some periodic "dips" in the graph as in the Figure 3.

○ Meter concentration problem. These problems are easy to find considering PRIME availability of meter concentrations, and may be caused by concentration noise.

- Line problem, detected by analysing the graph of PRIME availability ordered by its location as it is shown in the Figure 7.

- Phase problem, normally caused by a noise affecting only to one phase. In this type of problems, meters in a phase usually have worse performance than other meters in the same line but in different phases.

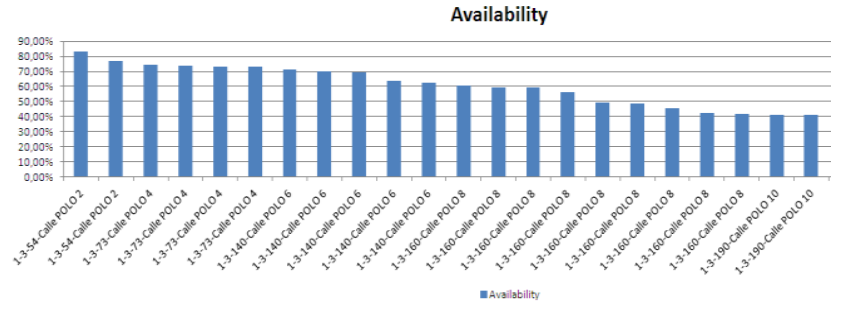

Figure. 7. Example of a line problem

- Transformer level problem. Normally because an auxiliary node [14] is needed but it is not communicating properly or its installation is incorrect.

- Individual problem of a meter such as incorrect firmware version or a bad installation.

- Manufacturer related problem. It is caused due to software issues, or hardware limitations.

- Application level:

- General problem of the network, normally caused by a DLMS problem in the concentrator or in all meters, or by a problem with the concentrator firmware version.

- Individual problem of a meter or a group of them. Normally the meters do not respond to the requests performed by the concentrator, or they respond with unexpected data.

- Database connectivity consistency:

- PRIME is an adequate way to detect the electrical connectivity, because smart meters register to the Base Node connected to its SS. Comparing field results with expected results (customer database information registered by the company), database consistency can be checked and corrected if needed. Even more, with appropriate hardware in the SS, it is possible to identify the LV line and phase each meter is connected to (phase is provided by means of zero-cross measurements).

- SS Categorization: Once we have enough networks deployed and analyzed, it is possible to categorize them and predict (always considering possible deviations caused by particular problems of the network) expected behavior of each network taking into account its electrical grid characteristics. For these purposes, two electrical parameters are used: number of Points of Supply (meters in the network) and concentration level of these meters in CGP's, also called density of Points of Supply [15]. The categorization with these two parameters is shown in Table II: 
Table II.- Categorization of SS's

\begin{tabular}{|c|c|c|c|c|}
\hline & \multicolumn{3}{|c|}{ Density of Points of Supply (DPoS) } \\
\hline & & $\begin{array}{c}0<\mathrm{DPoS} \\
<=6\end{array}$ & $\begin{array}{c}6<\mathrm{DPoS} \\
<=12\end{array}$ & $\begin{array}{l}0<\mathrm{DPoS} \\
<=6\end{array}$ \\
\hline \multirow{3}{*}{ 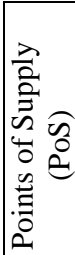 } & $\begin{array}{l}0<\mathrm{PoS} \\
<=100\end{array}$ & Category $\mathrm{G}$ & Category A & Category B \\
\hline & $\begin{array}{l}100<\mathrm{PoS} \\
<=300\end{array}$ & Category $\mathrm{H}$ & Category $\mathrm{C}$ & Category D \\
\hline & $300<\mathrm{PoS}$ & Category I & Category E & Category $\mathrm{F}$ \\
\hline
\end{tabular}

\section{Special features of the analysis}

The analysis performed during the deployment is used to identify special undesired events within a subnetwork.

One of them is cyclic noise, which can be located:

1) Globally, in the concentrator under analysis.

2) In the concentrator under analysis and thus in all meters of its subnetwork.

3) In one or more lines, CGP's, addresses or phases, affecting only to devices located there.

The noise is usually caused by different devices connected to the grid, which may introduce variations in noise levels and impedances, affecting directly to the SNR. Because of that, Service Nodes suffer disconnections, which can be of different durations depending on the characteristics of the perturbation.

Using PRIME Subnetwork Evolution graph in the analysis tool, it is possible to identify this type of problems easily. Figure 3 shows an example of this cyclic noise.

Once the affected devices are identified, it is necessary to find the noise source to solve the problem. To help with this work, the analysis tool generates a table that shows the likely electrical topology of the subnetwork. In this table, CGPs are ordered by their average availability, assuming that the better availability, the closer to the concentrator is the CGP. Figure 8 shows an example of this table where each row represents each LV electricity line of the grid.

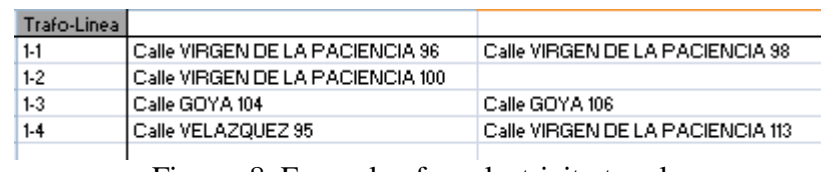

Figure. 8. Example of an electricity topology

PRIME protocol is an open solution, and many manufacturers can take part in the deployment. Experience has shown that is recommended to compare the behavior among devices of different manufacturers. With this purpose, the analysis tool generates a table which shows device availabilities grouped by manufacturer and meter concentration, so that meter manufacturer different performances in the same location can be evident. Figure 9 displays an example of this table.

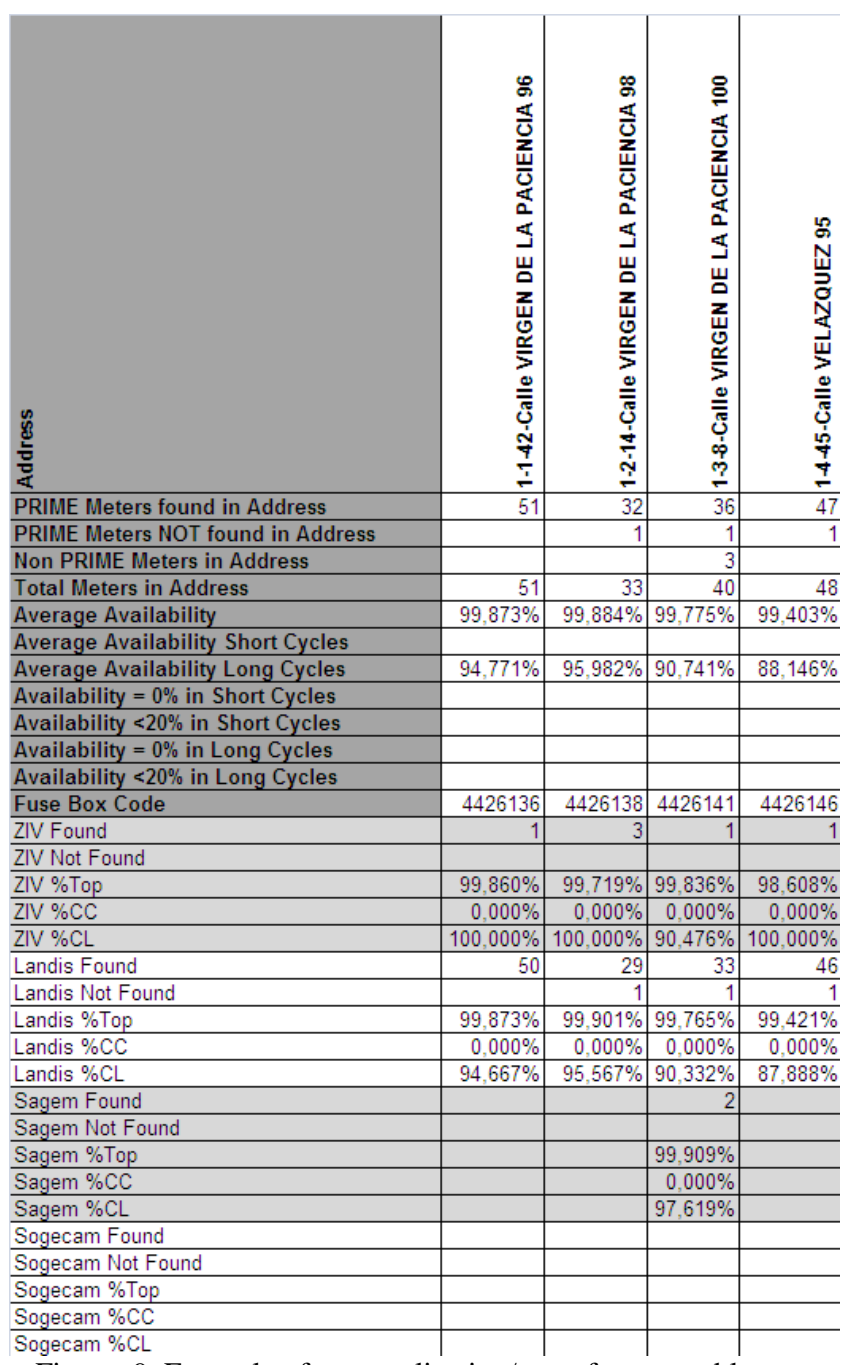

Figure. 9. Example of a centralization/manufacturer table

\section{Conclusions}

This paper has explained the need to analyse the results obtained in the deployment of Smart Metering networks based on PRIME specifications. The paper has also covered the typical performance analysis made for all deployed SS's, highlighting the different problems that might be found in this kind of deployment, and giving insights on its origins.

\section{References}

[1] Real Decreto 809/2006, [Electronic]. http://noticias.juridicas.com/actual

[2] "Informe 23/2009 de la CNE Solicitado por la Secretaria de Estado de Energía Sobre la Propuesta de Orden por la que se Establece el: "Plan Contador"", Comisión Nacional de Energía, 20 Jul. 2009, [Electronic], www.cne.es/cne/doc/publicaciones/cne119_09.pdf.

[3] Boletín Oficial del Estado, 21 Feb. 2012, [Electronic], http://www.boe.es/boe/dias/2012/02/21/pdfs/BOE-A2012-2538.pdf

[4] A. Zaballos, A. Vallejo, M. Majoral, and J.M. Selga, "Survey and Performance Comparison of AMR Over PLC Standards", IEEE Transactions on Power Delivery, Volume: 24, Issue: 2, 009, pp. $604-613$.

[5] C. Nunn, P.M. Moore, and P.N. Williams, "Remote meter reading and control using high-performance PLC 
communications over the low voltage and medium voltage distribution networks", Seventh International Conference on Metering Apparatus and Tariffs for Electricity Supply, 1992, pp. $304-308$.

[6] A. Hosemann, "PLC Applications in Low Voltage Distribution Networks", ISPLC 1997, Essen, Germany, Apr. 1997, pp. $134-139$.

[7] B.S. Park, D.H. Hyun, and S.K. Cho, "Implementation of AMR system using power line communication", IEEE/PES Transmission and Distribution Conference and Exhibition 2002: Asia Pacific. Volume: 1, pp. 18 - 21 vol. 1.

[8] EN 50065-1:2011, "Signaling in low-voltage electrical installations in the frequency range $3 \mathrm{kHz}-148.5 \mathrm{kHz}-$ Part 1: General requirements, frequency bands and electromagnetic disturbances", CENELEC, April 2011.

[9] A. Sendin, A. Llano, A. Arzuaga and I. Berganza, "Field Techniques to Overcome Aggressive Noise Situations in PLC Networks", In proceedings of IEEE SPLC 2011.

[10] PRIME Alliance, "Draft Standard for Powerline Intelligent Metering Evolution". Version R1.3.6.

[11] IEC 61334-4-32, "Distribution automation using distribution line carrier systems", Part 4, Section 32, September 1996.

[12] Real Decreto 842/2002, [Electronic]. http://noticias.juridicas.com/base_datos/Admin/rd842-

2002.html

[13] PRIME Alliance [Electronic]. http://prime-alliance.org/

[14] A. Sendin, R. Guerrero, P. Angueira, "Signal Injection Strategies for Smart Metering Network Deployment in Multitransformer Secondary Substations", IEEE Transactions on Power Delivery, Vol.26, No. 4, October 2011.

[15] A. Sendin, I. Berganza, A. Arzuaga, A. Pulkkinen, e I.H. Kim, "Performance Results from 100,000+ PRIME Smart Meters Deployment in Spain", 3rd IEEE International Conference on Smart Grid Communications (3rd SmartGridComm 2012), Tainan, Taiwan, November 2012. 